\title{
Emergency Department Chief Complaint Versus Discharge Diagnosis for Tracking Disease Measures
}

\author{
Andrea Bankoski, Anikah Salim and Zachary Faigen* \\ Maryland Department of Health and Mental Hygiene, Baltimore, MD, USA
}

\section{Objective}

The state of Maryland has incorporated chief complaint data from $100 \%$ of its acute care emergency departments (ED) into the Electronic Surveillance System for the Early Notification of Community-Based Epidemics (ESSENCE). In 2012, the Maryland Department of Health and Mental Hygiene (DHMH) began using this statewide disease surveillance system to track several specific disease measures including certain chronic diseases. The validity of using ESSENCE ED data to track and analyze these health outcomes was evaluated.

\section{Introduction}

ESSENCE is a web-based syndromic surveillance system utilized by DHMH to detect and track outbreaks, suspicious patterns of illness, public health emergencies, and biological threats. ESSENCE ED chief complaint data is collected daily from 47 emergency departments in Maryland (all 45 acute care hospitals and 2 freestanding emergency medical facilities). A chief complaint in ESSENCE is a free text field that lists the patient's reason for the ED visit upon arrival at the hospital. Chief complaint data may be comprehensive or abbreviated and may include a single reason or multiple reasons for the ED visit. Medical history may be included in chief complaint data, which can create low specificity (false positive cases). Chief complaint data alone may yield less accurate modeling and lower outbreak detection sensitivity. This analysis evaluates whether counts of chief complaints are appropriate indicators of disease burden for several specific illnesses, by comparing chief complaints to their corresponding discharge diagnoses.

\section{Methods}

ESSENCE data from 2012 was queried for six measures - alcohol intoxications, asthma, congestive heart failure (CHF), dental issues, diabetes, and attempted suicides. Each measure contained one complete year of data, excluding alcohol intoxications, which contained six months of data. Alcohol intoxications, CHF, and attempted suicides were reviewed for all ages, while asthma was reviewed only for 0 to 17 year olds and diabetes was reviewed for adults 20 years of age and older. A comparison was made between free text discharge diagnosis data currently collected from 8 of the 47 emergency departments and chief complaint data. For a chief complaint to be considered valid, the corresponding discharge diagnosis had to contain a keyword for the measure alone (alcohol, asthma, CHF/heart failure, suicide/ suicidal, diabetes, dental) or in combination with other words.

\section{Results}

For all measures, a substantial percentage of chief complaints did not match the corresponding discharge diagnoses: attempted suicides $(51.7 \%)$, asthma (37.2\%), diabetes (33.6\%), alcohol intoxication (21.2\%), dental issues (19.8\%), and CHF (17.6\%). On average, $30.2 \%$ of the chief complaints did not match discharge diagnoses. It appears that chief complaint data for certain disease conditions do not accurately represent the reason for the ED visit. However, only 8 out of $47(17.0 \%)$ hospitals were used in this analysis due to the availability of the discharge data. Further analysis will be conducted once more hospitals provide discharge data.

\section{Conclusions}

Until more hospitals provide discharge data, DHMH has decided that ED chief complaint counts from ESSENCE that represent specific diseases or conditions should not be used for tracking or other surveillance purposes. However, ESSENCE will continue to be used for tracking syndromes (such as fever, gastrointestinal, neurological, respiratory, etc.) by use of its internal algorithms in order to identify abnormally high daily counts in comparison to counts from previous days.

\section{Keywords}

chief complaint; discharge diagnosis; ESSENCE; chronic disease; surveillance

\section{Acknowledgments}

The Office of Preparedness \& Response at DHMH would like to acknowledge and thank the Vital Statistics Administration and the Virtual Data Unit at DHMH.

*Zachary Faigen
E-mail: zachary.faigen@maryland.gov 